\title{
Nanoleakage for Self-Adhesive Resin Cements used in Bonding CAD/CAD Ceramic Material to Dentin
}

\author{
Wafa El-Badrawy \\ Randa Mohamed Hafez \\ Abeer Ibrahim Abo El Nagac \\ Doaa Ragai Ahmed ${ }^{d}$
}

\section{ABSTRACT}

Objectives: To determine nanoleakage of CAD/CAM ceramic blocks bonded to dentin with selfadhesive resin cement.

Methods: Eighteen sound extracted human molars were sterilized and sectioned into $3 \mathrm{~mm}$-thick dentin sections. Trilux Cerec Vitablocks (Vita) were also sectioned into $3 \mathrm{~mm}$ sections, surfacetreated using $5 \%$ hydrofluoric acid-etchant, and then coated with silane primer (Vita). Trilux and dentin sections were cemented together by means of three resin cements: Rely-X Unicem (3M/ ESPE), BisCem (Bisco), and Calibra (Dentsply), according to manufacturers' recommendations. Calibra was used in conjunction with Prime/Bond-NT adhesive (Dentsply), while the other two are self-adhesive. The bonded specimens were stored for $24 \mathrm{~h}$ in distilled water at $37^{\circ} \mathrm{C}$. Specimens were vertically sectioned into $1 \mathrm{~mm}$ - thick slabs, yielding up to six per specimen. Two central slabs were randomly chosen from each specimen making up the cement groups $(n=12)$. Each group was subdivided into two subgroups $(n=6)$, a control and a thermocycled subgroup $\left(5-55^{\circ} \mathrm{C}\right)$ for 500 cycles. Slabs were coated with nail polish up to $1 \mathrm{~mm}$ from the interface, immersed in a $50 \%$ silver nitrate solution for 24h, and tested for nanoleakage using Quanta Environmental SEM and EDAX. Data were statistically analyzed using two-way ANOVA and Tukey's post-hoc tests.

Results: Rely-X Unicem and Calibra groups demonstrated no significant difference in the percentage of silver penetration, while the BisCem group revealed a significantly higher percentage ( $P \leq$.05). Thermocycling (500 cycles) did not have a statistically significant effect on the percentage of silver penetration $(P>.05)$.

Conclusions: One self-adhesive-resin cement demonstrated a similar sealing ability when compared with a standard resin cement. Thermo-cycling did not significantly increase dye penetration under the test conditions. (Eur J Dent 2011;5:281-290)

Key words: Bonding; Nanoleakage; Self-adhesive; Ceramic blocks; CAD/CAM.

a Department of Clinical Sciences, Restorative Discipline, Faculty of Dentistry, University of Toronto, Toronto, Canada.

b Department of Operative Dentistry, Faculty of Oral and Dental Medicine, Cairo University, Cairo, Egypt.

Department of Operative Dentistry, College of Oral and Dental Surgery, Misr University for Science and Technology, Misr, Egypt. d Department of Operative Dentistry, Faculty of Oral and Dental Medicine, Alexandria University, Alexandria, Egypt.

Corresponding author: Dr. Wafa El-Badrawy Department of Clinical Sciences, 124 Edward Street, Toronto, rm \# 352D M5G 1G6, Ontario, Canada Phone: 4169794934 ex. 4343 Fax: 4169794936 E-mail: w.badrawyldentistry.utoronto.ca 


\section{INTRODUCTION}

The use of bonded ceramic restorations in dentistry has recently gained widespread popularity. The primary reason, among others, for this increased popularity is their contribution to overcoming some of the problems associated with direct composite restorations. One of these major problems involves shrinkage stresses that occur during the polymerization ${ }^{1,2}$ of direct resin composites, with subsequent cuspal distortion, postoperative sensitivity, marginal discrepancies, and microleakage. ${ }^{3-5}$ Esthetic indirect restorations limit the contraction stresses to the resin cement layer alone. ${ }^{6,7}$ Nonetheless, the resulting toothcement-restoration interface consequently poses a new challenge during the cementation procedure, accompanied by new, associated problems.

Despite the significant improvements that have been made to adhesive systems, the bonded interface continues to remain the weakest point. Ideally, the adhesive would allow restorative material bio-integration and provide strong and durable bonds that would seal the tooth-restoration interface, preventing microleakage and the subsequent ingress of microorganisms. 8,9 The introduction of a new generation of bonding systems and contemporary adhesive cements generates hope that such bonds to the tooth structure as well as the indirect restorations can now be obtained. ${ }^{10}$

Self-adhesive resin cements were recently developed, with the rationale of simplifying the cementation procedure by eliminating the pretreatment step involving the tooth structure. This development has presented a rather promising prospect, due to shortened application times and the subsequent decreased technique sensitivity. ${ }^{11}$ However, the new self-adhesive resin cements do not necessarily guarantee equal or improved bonding efficacy. In laboratory and clinical research, conventional bonding and cementation procedures have generally proven themselves to perform best, thus becoming the "gold standards". ${ }^{11,12}$

For many years, the clinical success of cemented restorations has been evaluated by measuring the marginal fit and the extent of microleakage. However, in 1994, Sano et al $^{13}$ described a new pattern of microleakage that occurs in the absence of gaps and which results from subsurface porosity of demineralized dentin. This form of leakage was detected on a microscopic, as opposed to a macroscopic level, and was thus referred to as "nanoleakage."
This nanoleakage may be the result of residual water around collagen fibrils, collapse of the collagen network, or incomplete resin infiltration into exposed collagen network and polymerization. ${ }^{14}$ Although the amount of nanoleakage may be very small (nanometer-size) in the bonded assembly, over time, it has the potential to serve as a pathway for water movement within the adhesive-resin interface. Such water movement may extract unconverted monomers from the resin adhesive or hybrid layer. Therefore, the effect of nanoleakage upon the integrity of resin-dentin bonding has become an issue of interest, not only for short-term, but for long-term adhesion in particular..$^{15}$ The formation of nanoleakages within the hybrid layer and the adhesive-resin interface is an important indicator for judging the material's sealing ability and for the quality of the hybrid layer, which, in turn, affects the longevity of the restoration. ${ }^{16}$

The bond durability of self-etching bonding systems have been heavily investigated by several researchers, including $\mathrm{Li}$ et $\mathrm{al},{ }^{17}$ Mitsui et al, ${ }^{18}$ and Pneumans et al, ${ }^{12}$ who concluded that alletch systems perform better and have increased longevity. However, little information is available regarding nanoleakage of self-adhesive resin cements to prefabricated indirect restorations and the durability of the bonds. Therefore, the purpose of this study was to determine the nanoleakage pattern of resin cements used with indirect CAD/ CAM restorations.

The objectives of this study were to examine the ultrastructure of the bonded interface using scanning electron microscopy and to compare the sealability of self-adhesive resin cements to that of a total etch resin cement by examining the nanoleakage pattern along the resin dentin interface.

\section{MATERIALS AND METHODS}

Eighteen extracted, caries-free human molars were cleaned, examined, and sterilized using a $0.5 \%$ chloramine $T$ solution. The teeth were stored under refrigerated conditions and were used within a maximum of one month following their extraction.

The occlusal third of the crown of each tooth was removed by sectioning the crown perpendicular to its long axis using a low speed diamond saw (Buehler- Isomat, LakeBulff, IL, USA) under copious amounts of water. An additional section was formed $1 \mathrm{~mm}$ above the cemento-enamel junction in order to remove the roots and obtain a circular 
dentin section approximately $3 \mathrm{~mm}$ in thickness. The dentin surfaces were ground on a polishing machine using 600-800 grit silicon carbide papers in order to create a standard smear layer in preparation for bonding to Cerec blocks.

Triluxe Vitablocks, designed for the Cerec CAD/CAM system (Vita Zanfabrik, Bad Sackingen, Germanyl were used. Eighteen ceramic sections ( $3 \mathrm{~mm}$ - thick) were obtained by sectioning the blocks using the low speed diamond saw described above. Triluxe sections' surfaces were prepared for bonding by an acid etching process using 5\% hydrofluoric acid (Vita Zanfabrik, Bad Sackingen, Germanyl for 60 seconds. They were then washed, dried and primed using silane ceramic primer (Vita Zanfabrik, Bad Sackingen, Germanyl for 15 seconds, followed by air drying to allow complete evaporation of the solvent.

Both dentin and ceramic sections were randomly divided into three groups according to the type of resin cement used for bonding. Cement types, composition, and manufacturers are shown in Table 1. Sections from groups I and II were bonded using self-adhesive resin cements (Rely-X and BisCem, respectively) where no pretreatment for the dentin sections' surfaces was carried out. Group III dentin sections, however, were conditioned prior to bonding according to the manufacturer's instructions of Prime \& Bond NT (Dentsply, Caulk, Milford, Del, USA). In all groups, the mixing and application of the resin cements and bonding procedures were performed according to the manufacturers' recommendations. Treated sections were bonded together and light cured for two seconds initially, allowing for removal of any excess cement. Each side of the bonded specimens was then light-cured for an additional 20 seconds using an Optilux light curing unit (SDR, Kerr, USA). All the bonding procedures were carried out at room temperature. The bonded specimens were then stored in distilled water at $37^{\circ} \mathrm{C}$ for 24 hours.

Bonded specimens were then vertically sectioned into several $1 \mathrm{~mm}$-thick slabs perpendicular to the adhesive interface ${ }^{19}$ using a low speed diamond saw (Isomet, Buehler Ltd, Lake Bluff, IL, USA). Two central slabs were chosen from each bonded specimen, forming a total of 12 slabs per group. Sectioned slabs of each group were further subdivided into 2 subgroups $(n=6)$ : a control subgroup and a thermocycled subgroup. Thermocycling was conducted in water that was between 5 and $55^{\circ} \mathrm{C}$ for 500 cycles (with 20 seconds dwell time).
All of the bonded slabs were then coated with two layers of fast-drying nail varnish applied 1 $\mathrm{mm}$ away from the bonded interfaces. ${ }^{20}$ Following the drying of the nail varnish coats, specimens were immersed in distilled water for 10 minutes in order to allow for rehydration prior to immersion in a freshly prepared silver nitrate tracer solution for 24 hours in light proof containers. The silver nitrate solution $(50 \%)^{13}$ was prepared by dissolving 50 grams of pure silver nitrate in 100 $\mathrm{ml} / \mathrm{L}$ of distilled water. The specimens were then thoroughly rinsed using distilled water and immersed in a photodeveloping solution for 8 hours under a fluorescent light.

Slabs were wet-polished using three descending orders of Sof-Lex discs (medium, fine and ultra-fine), cleaned ultrasonically and immersed in $6 \mathrm{~mol} / \mathrm{L} \mathrm{HCl}$ for 30 seconds to remove the polishing smear layer..$^{13}$ The slabs were then examined using a Quanta Environmental Scanning Electron Microscope (QESEM) (Philips, France) to determine the degree of silver deposition within the formed hybrid layer and within the resin/dentin interface. Further analysis of the surfaces using Electron Dispersive Analytical X-ray (EDAX) was carried out in order to obtain a quantitative analysis regarding the elemental composition of the examined specimen.

Means and standard deviations of the percentages of silver penetration data were calculated for each group and were statistically analyzed using Statistical Package for Scientific Studies for Windows SPSS 15.0 (SPSS Inc., Chicago, IL, USA). A regression model with a two-way analysis of variance (ANOVA) and Tukey's post-hoc test $(P \leq .05)$ were used.

\section{RESULTS}

Means and standard deviations of silver penetration percentages (as indicated by EDAX) of the total examined interface for the different groups are shown in Table 2 and Figure 1. As shown in the results, silver deposition was present in all experimental groups, with the BisCem group demonstrating the highest means of silver penetration and the Calibra group showing the lowest. Tukey's test indicated that the process of thermocycling had no statistically significant effect on the silver penetration percentages of all subgroups (P>.05). Meanwhile, both BisCem resin cement groups showed statistically significant higher mean silver penetration percentages compared to the RelyX and Calibra groups, with 
no significant difference being demonstrated between the latter two groups (Table 2 and Figure 1).

Figures 2-7 represent SEM micrographs along with their corresponding EDAX spectrum. In each of the micrographs, many shiny spots were observed. These spots, however, did not correspond with increased silver percentages when analyzed using EDAX. In fact, these shiny areas were found

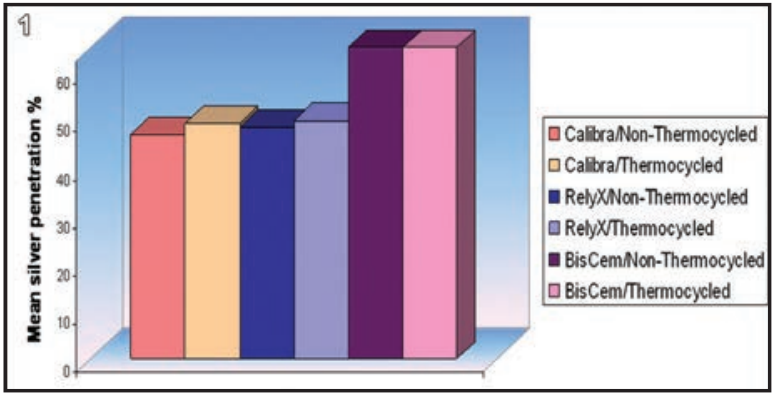

Figure 1. Means of silver penetration values in all subgroups.
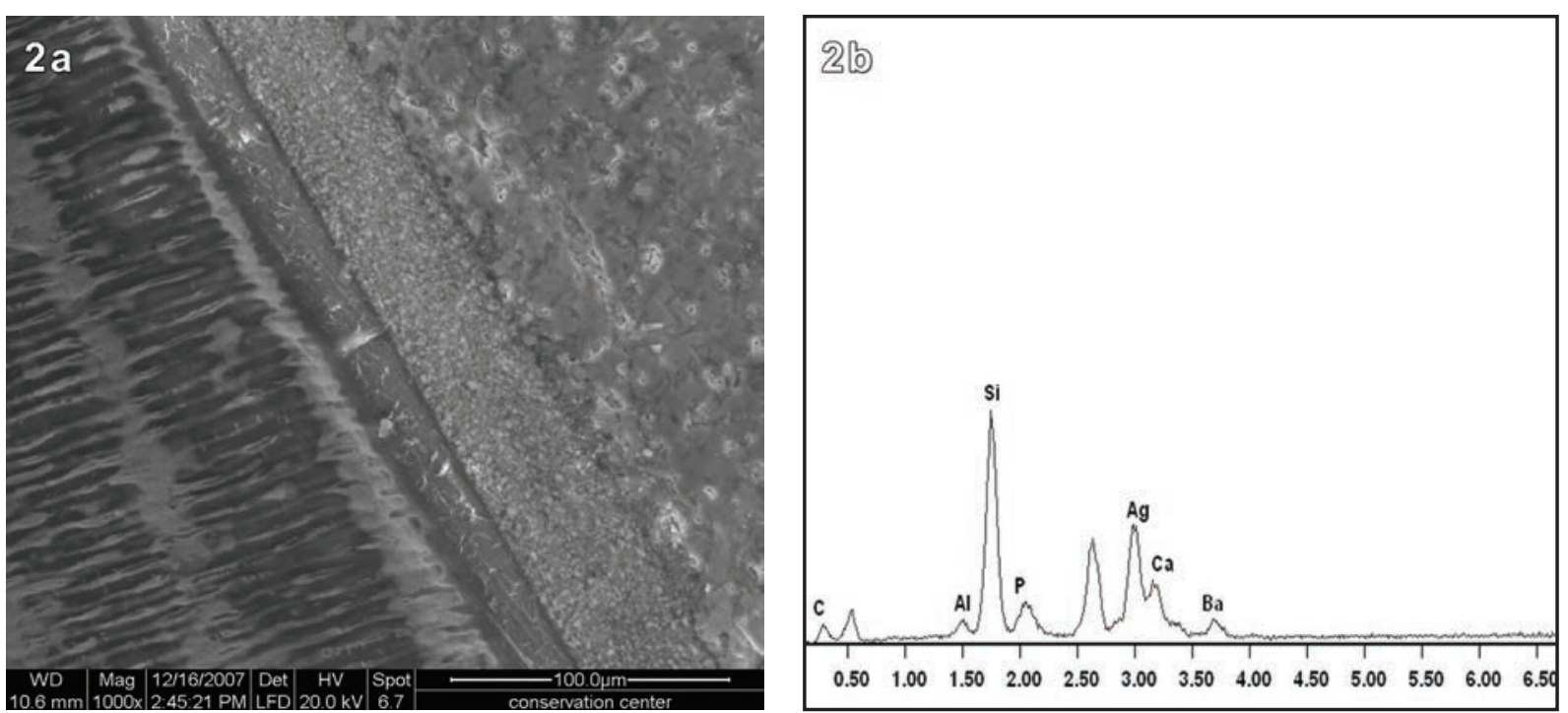

Figure 2. a. SEM micrograph of non-thermocycled Calibra Resin Cement and b. its corresponding EDAX spectrum curve at one point along the tooth/restoration interface.
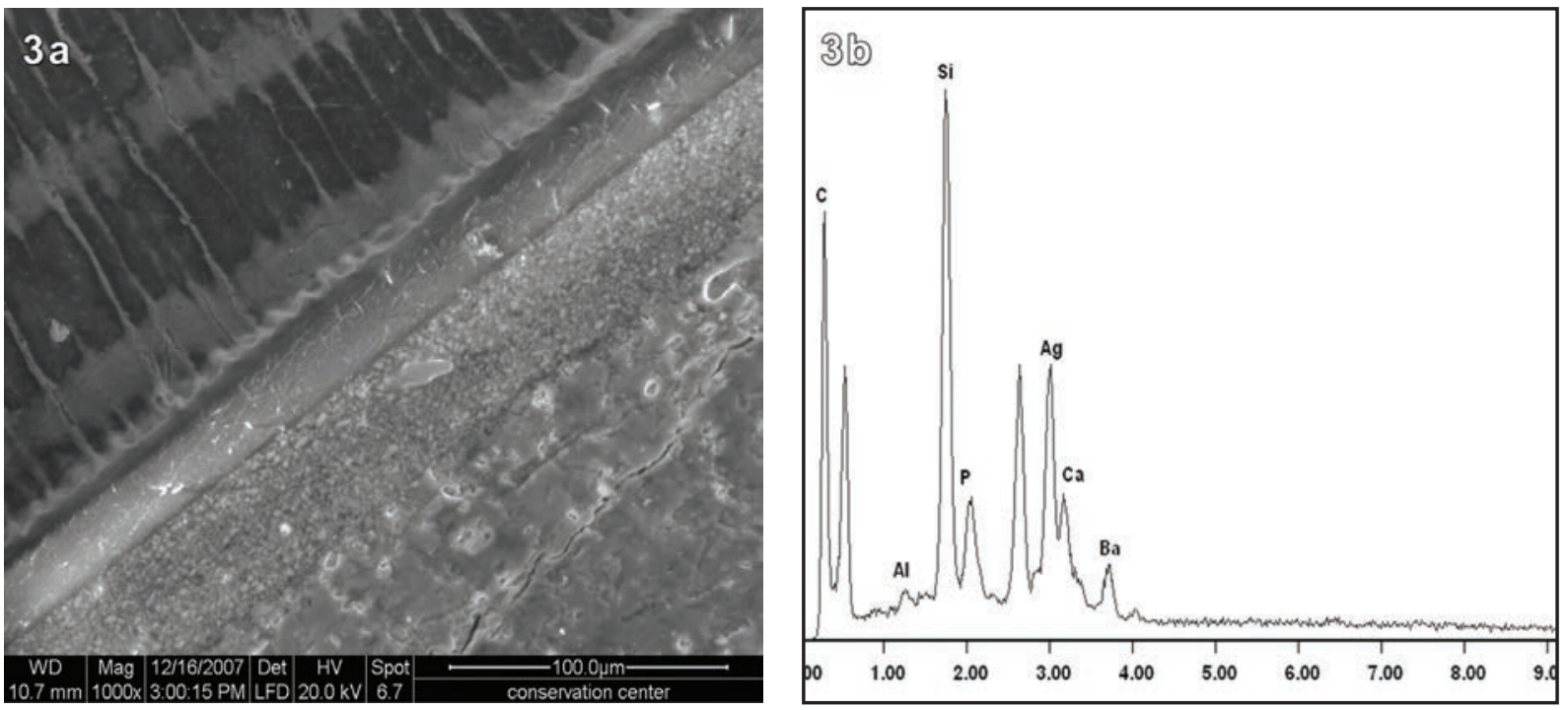

Figure 3. a. SEM micrograph of thermocycled Calibra Resin Cement and b. its corresponding EDAX spectrum curve at one point along the tooth/restoration interface.

to be completely free of silver and were comprised only of silica and calcium. The ultrastructure of Calibra resin cement (Figures 2 and 3) generally showed a distinctive hybrid layer with long resin tag formation, while in the RelyX group (Figures 4 and 5), shorter resin tags were observed. In the case of the BisCem group, the scanning micrographs (Figures 6 and 7) showed an absence of resin tag formation. Dark areas were present in Figures 4-7, which appeared to be gaps formed at the interface between dentin and the ceramic surface. These gaps were present when the specimens were observed using the large field laser beam. However, when using the backscattered beam (Figure 8), it became apparent that these dark areas were filled with the adhesive, confirming that no gap was present.

European Journal of Dentistry 

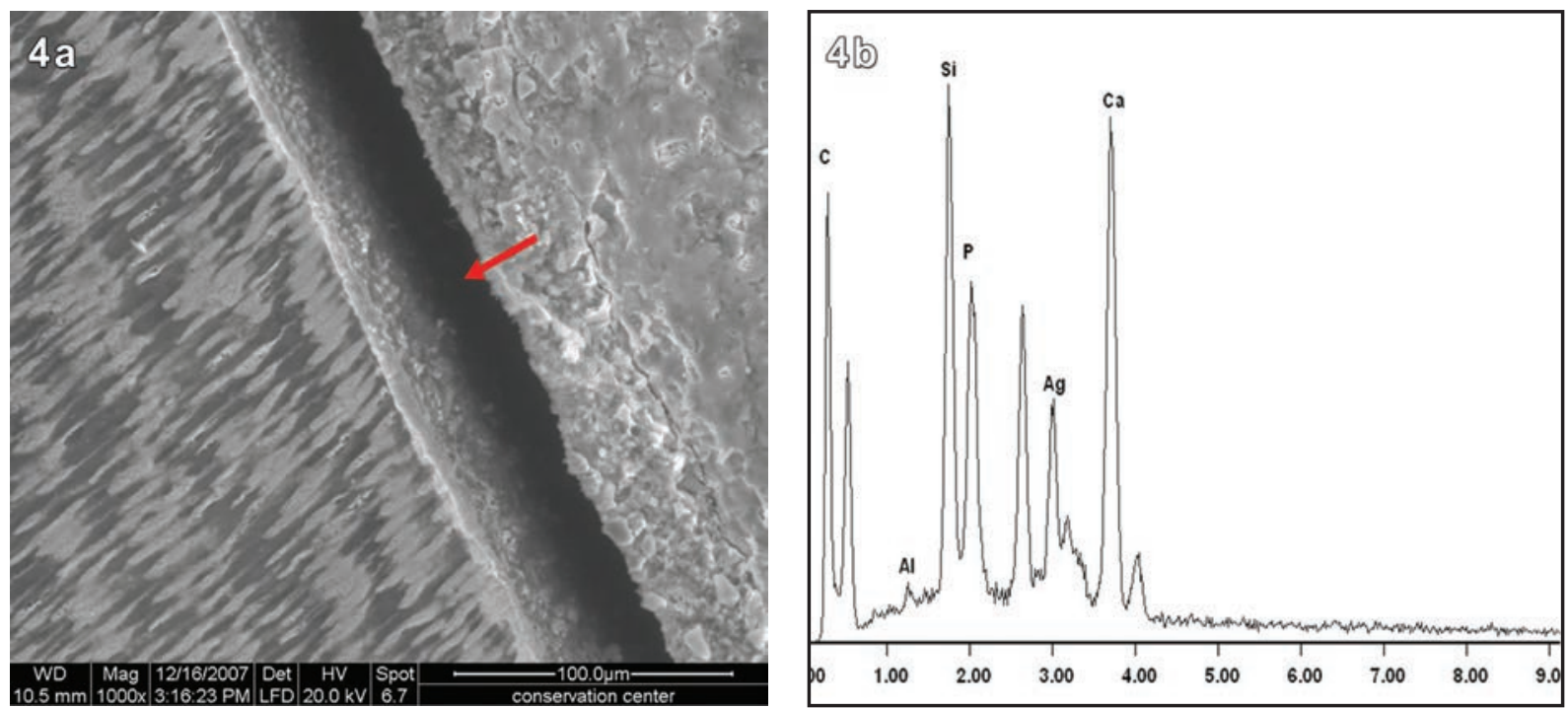

Figure 4. a. SEM micrograph of non-thermocycled RelyX Resin Cement and b. its corresponding EDAX spectrum curve at one point along the tooth/restoration interface. N.B. The arrow points to the adhesive layer.
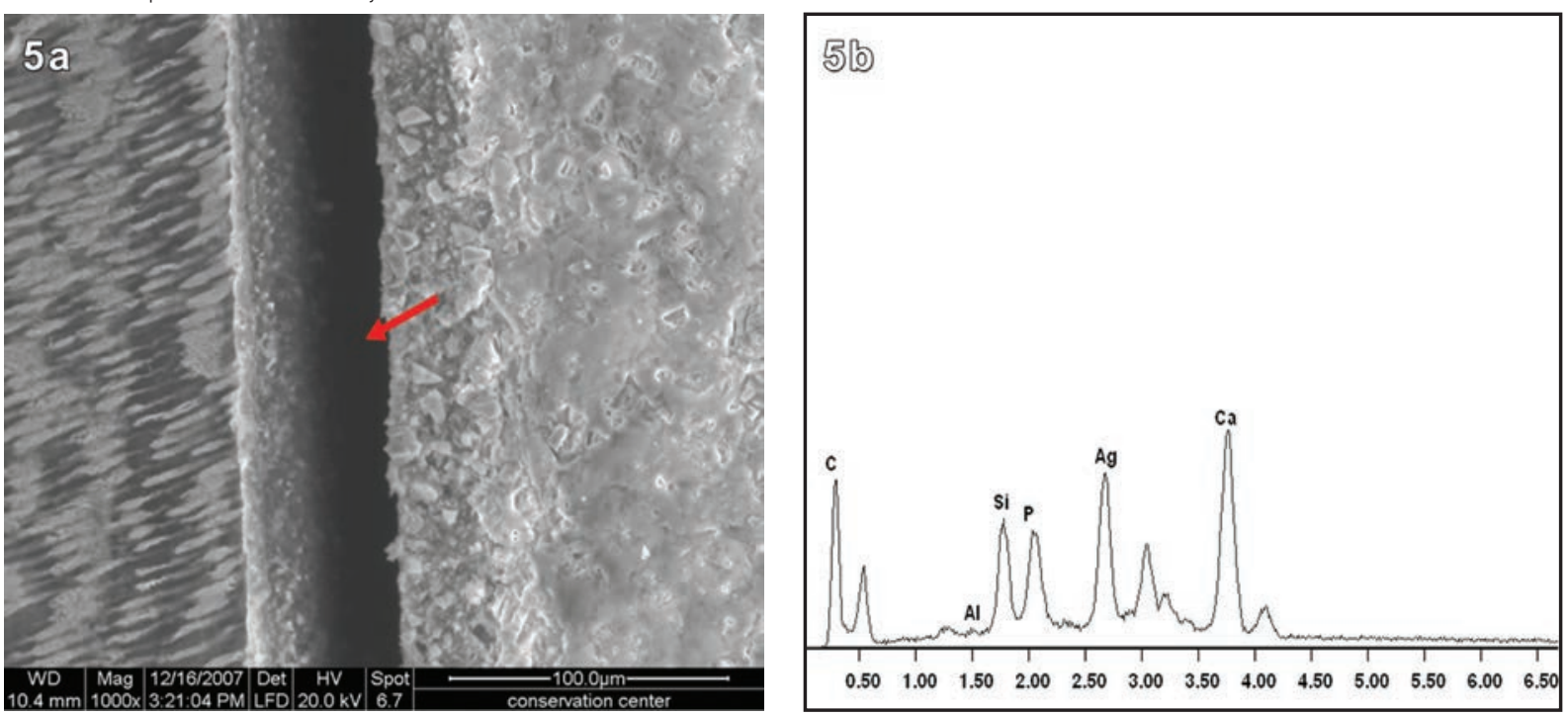

Figure 5. a. SEM micrograph of thermocycled RelyX Resin Cement and b. its corresponding EDAX spectrum curve at one point along the tooth/restoration interface. N.B. The arrow points to the adhesive layer.

Table 1. Manufacturers and compositions of adhesive materials used.

\begin{tabular}{lcc}
\hline Cement & Adhesive used & Manufacturer \\
\hline $\begin{array}{l}\text { RelyX Unicem } \\
\text { Dual-cured self-adhesive resin cement }\end{array}$ & None & 3M ESPE Dental Products St. Paul, USA \\
$\begin{array}{l}\text { BisCem } \\
\text { Dual-cured self-adhesive resin cement }\end{array}$ & None & Bisco, Inc. Schaumburg, IL 60193, USA \\
$\begin{array}{l}\text { Calibra Esthetic } \\
\text { Dual-cured resin cement system }\end{array}$ & Prime \& Bond NT & Dentsply Caulk, Milford, DE, USA \\
\hline
\end{tabular}

Table 2. Means and standard deviations of silver nitrate percentages of all tested thermocycled and non-thermocycled resin cements.

\begin{tabular}{lccc}
\hline Variables interaction & Mean & SD & P-value \\
\hline RelyX/Non-Thermocycled & $48.4 \mathrm{~b}$ & 27.8 & 6.6 \\
BisCem/Non-Thermocycled & $85.1 \mathrm{a}$ & 19.3 & $0.007^{*}$ \\
Calibra/Non-Thermocycled & $46.8 \mathrm{~b}$ & 24.5 & 3.2 \\
RelyX/Thermocycled & $49.8 \mathrm{~b}$ & 22.5 & \\
BisCem/Thermocycled & $87.9 \mathrm{a}$ & & \\
Calibra/Thermocycled & $49.1 \mathrm{~b}$ & &
\end{tabular}

July 2011 - Vol.5 


\section{DISCUSSION}

The Quanta Environmental Scanning Electron Microscope (QESEM) used in this study is a recently introduced analytical tool that provides exceptional depth of field and allows for minimal specimen preparation, allowing examination of the specimens without a gold or carbon coating. The concurrent EDAX analysis was carried out in order to identify the existence of metallic silver particles. The use of SEM in conjunction with EDAX enables distinct images to be captured together with sensitive and accurate analysis. ${ }^{19}$ When SEM was used alone for nanoleakage examinations, with secondary and backscattered electron imaging, ${ }^{13,16}$ erroneous interpretations were common. ${ }^{21}$ EDAX, in contrast, provides accurate quantitative analysis and distribution for the various existing elements. The results of nanoleakage analyses were expressed in terms of percentages of silver deposition at three representing points; then the extent of silver penetration was calculated to determine the total examined interface that was penetrated by silver nitrate. ${ }^{13,16}$

Silver nitrate staining is the most commonly used material for nanoleakage evaluation as it easily migrates within the interface zone due to its extremely small diameter molecule $(0.059 \mathrm{~nm}){ }^{22}$ Moreover, silver nitrate induces an electron microscopic measurable contrast providing a sharp picture of the degree of penetration into the interface. Following its penetration, it has the potential to immobilize, which prevents further penetration during specimen preparation. ${ }^{17,21}$ Other organic dyes lincluding methylene blue, and basic fuch-
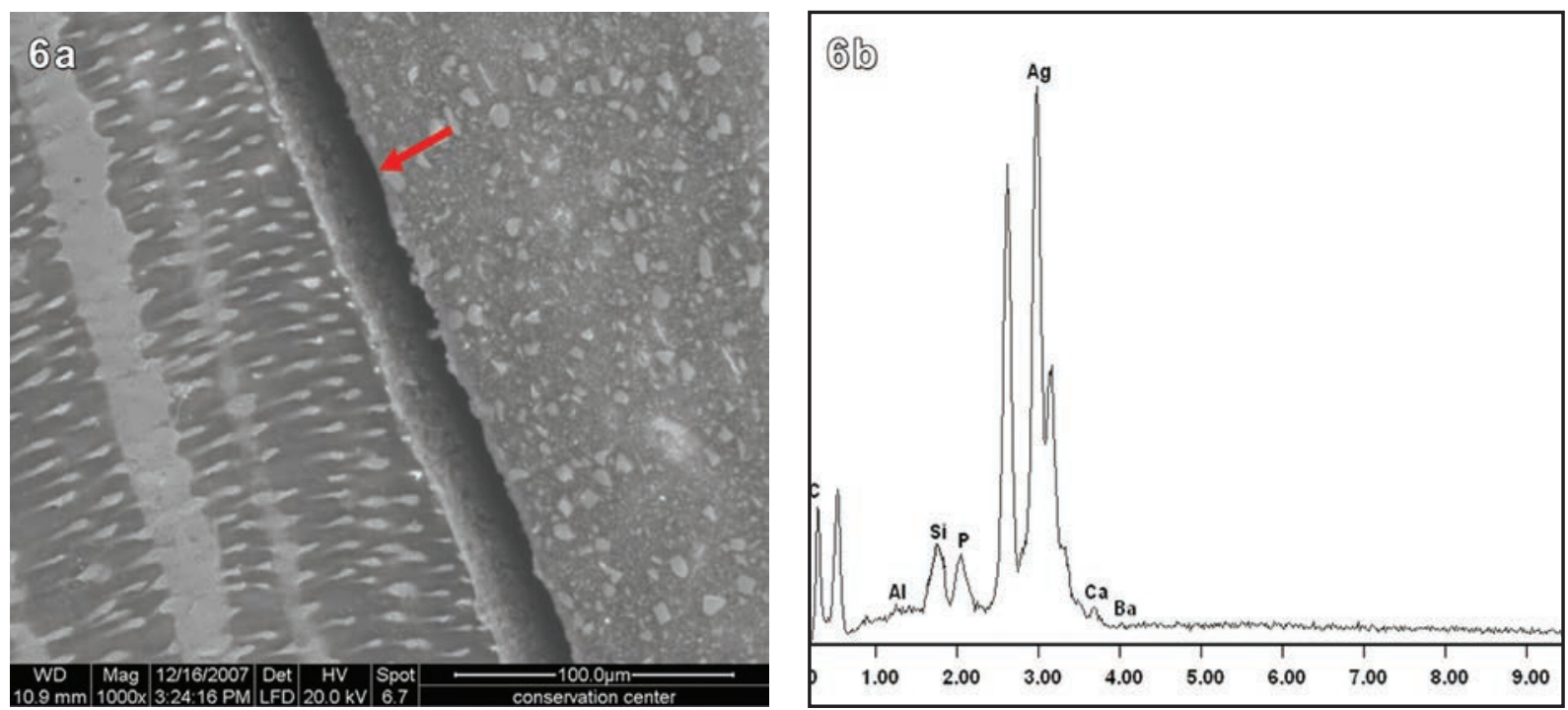

Figure 6. a. SEM micrograph of non-thermocycled BisCem Resin Cement and b. its corresponding EDAX spectrum curve at one point along the tooth/restoration interface. N.B. The arrow points to the adhesive layer.
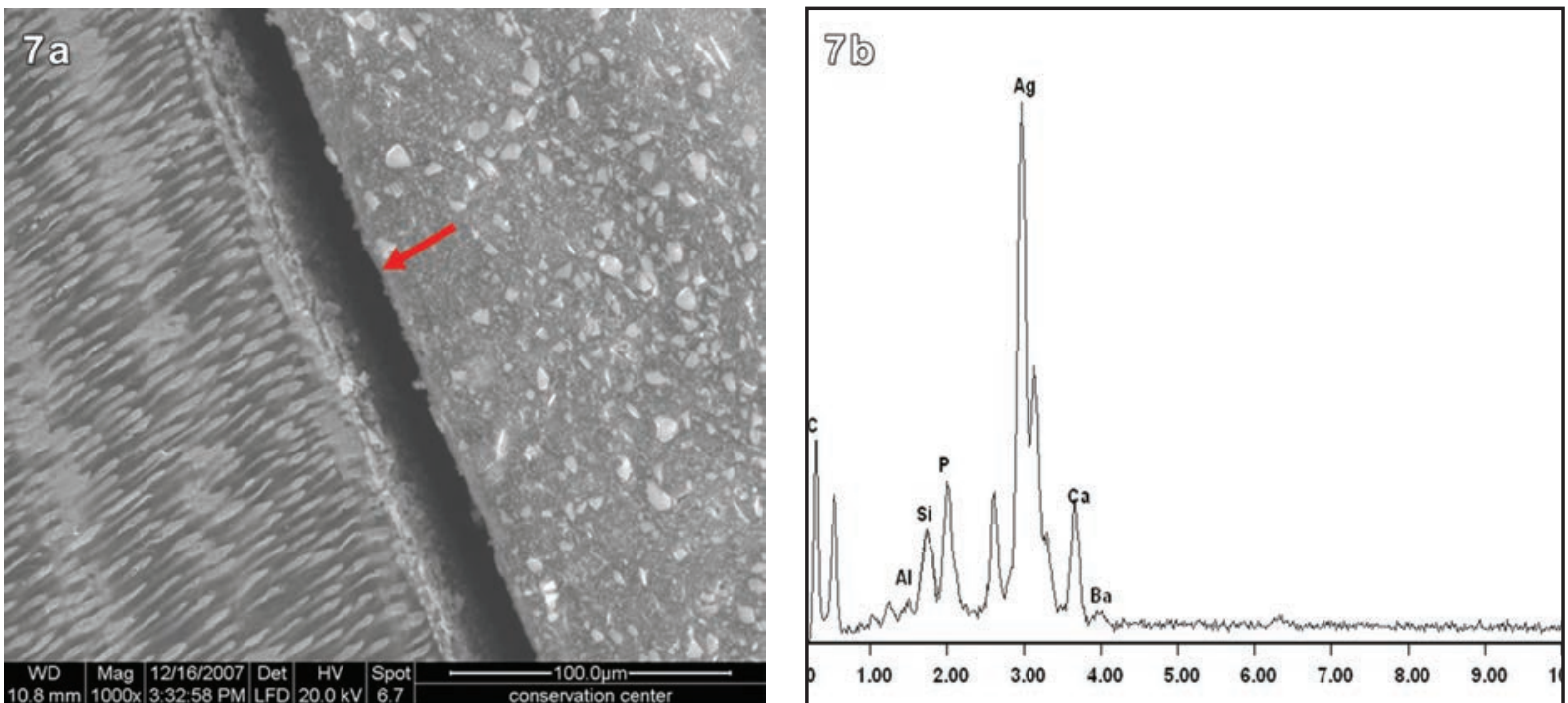

Figure 7. a. SEM micrograph of thermocycled BisCem Resin Cement and b. its corresponding EDAX spectrum curve at one point along the tooth/restoration interface. N.B. The arrow points to the adhesive layer.

European Journal of Dentistry 
sin) have been used for evaluation of leakage, but these substances have larger molecules and exhibit a propensity for bonding to tooth structure that can potentially demonstrate a wider and deeper gap than actually exists.

In order to evaluate the durability of the bond, the bonded specimens were subjected to thermocycling. The longevity of bonded restorations has long been evaluated by the use of a thermal cycling technique in both microleakage and nanoleakage studies. ${ }^{23}$ Thermocycling is applied in order to simulate oral conditions and to introduce an artificial aging parameter that would allow for the evaluation of temperature-dependent degradation of the materials. ${ }^{24}$ It has been reported that hot water may accelerate hydrolysis of the interface components, with subsequent water uptake and leaching of the breakdown products or poorly polymerized resin oligomers. In addition, thermal cycling may induce stresses between the tooth substrate and the restorative material. ${ }^{25}$ Such stresses can eventually lead to cracks that propagate along bonded interfaces, accelerating chemical degradation of the bonds.

Numerous investigations have employed this technique for providing laboratory simulations of oral conditions. The concept is to subject the bonded interface to the mechanical stresses generated by the different thermal conductivities and coefficient of the thermal expansion of the substrates and bonded materials. Thermocycling for 500 cycles $\left(5-55^{\circ} \mathrm{C}\right)$ was selected for this study. According to the ISO TR 11450 standard (1994), this regimen is considered an appropriate artifi-

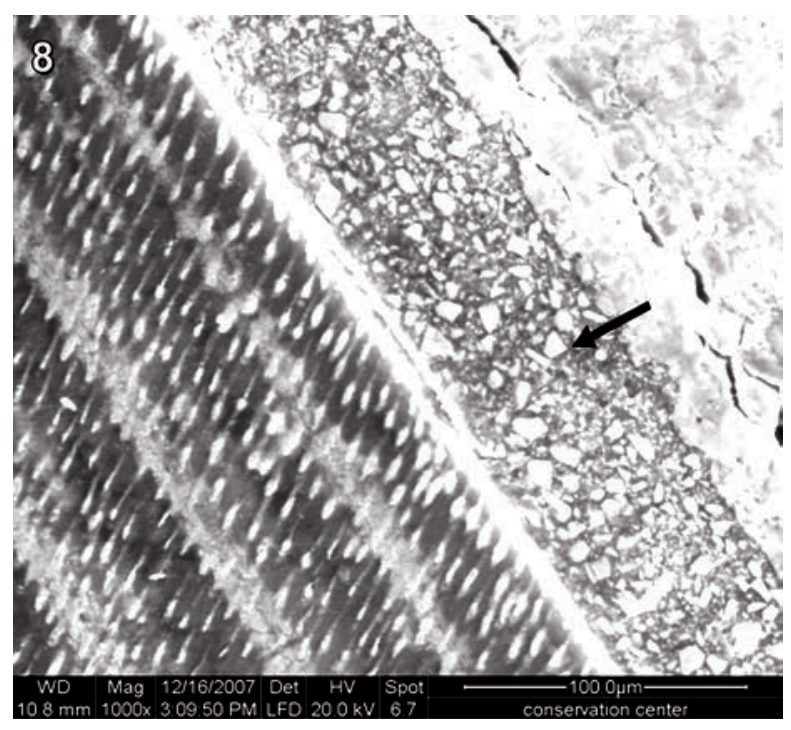

Figure 8. SEM micrograph of non-thermocycled RelyX Resin Cement, using backscattered beam to confirm the presence of the adhesive layer, which appeared as a gap when a large field laser beam was used. cial aging test. ${ }^{26}$

In the case of Calibra resin cement that was used in conjunction with a total etch adhesive resin, the presence of silver deposition could be attributed to insufficient adhesive resin infiltration/ penetration into the demineralized dentine. ${ }^{13,16}$ On the other hand, the occurrence of nanoleakage observed with the use of self-adhesive cements is probably due to a different scenario, since no previous demineralization of dentin is performed. In accordance with Tay et $\mathrm{al}^{27}$ and Hashimoto et $\mathrm{al}^{28}$ this could be attributed to the incomplete polymerization of the resin due to the presence of residual water, which is responsible for the silver uptake. Residual water may persist due to the lowering of vaporization in the presence of HEMA. Subsequently, HEMA copolymerizes with acidic resin monomers in the presence of water, forming homogenous hydrogels which permit fine silver deposits at the bonded interface. ${ }^{29}$

Regardless of the significant nanoleakage in all cements, the significantly higher silver penetration showed by BisCem in comparison to RelyX and Calibra cements suggests the inadequacy of the bond provided by this cement. This was also evident during specimen preparation, as bonded specimens were separating during slab sectioning. The fact that this batch of BisCem product was later recalled by the manufacturer raises questions regarding its results in the study. It is possible that the poor bond exhibited by this cement could likely be due to manufacturing problems. Alternatively, it may have resulted from weak selfadhesive ability of the resin cement. This weak ability may, in turn, have resulted in the absence of hybrid layer formation as revealed by the QESEM micrographs. Tay et al $(2002)^{30}$ and Tay et al $(2004)^{31}$ reported that the deficient seal of the self adhesive resin cements was probably due to transudation of dentinal fluid across these simplified adhesives, which was speculated to interfere with the proper polymerization of the luting resin cement by inducing emulsion polymerization of the coupling hydrophobic resin cement. It has also been reported that the slow-setting of the dual/ auto-cured resin cement allow sufficient movement of dentinal fluid sufficient to cause microblisters at the tooth/resin interface, which may result in subsequent failure of the bond. ${ }^{31}$ This may explain the nanoleakage seen in the above micrographs and the silver uptake within the interfaces, which can be attributed to a lack of frank resin tags in the self-adhesive cements. The lack of use 
of a separate primer may also have decreased the depth of infiltration of the adhesive resin.

In spite of the different chemistries and mode of bonding to dentin of Rely $X$ and Calibra cements they behaved almost similarly in terms of nanoleakage with no significant difference between them. This may be attributed to the claimed strong self-etching ability of the Rely $X$ resin cement (which has an initial low $\mathrm{pH}$ of $<2$, as reported by the manufacturer ${ }^{26}$ ). These results were in agreement with Behr et $\mathrm{al}^{32}$ and Ibarra et $\mathrm{al}^{26}$ who found that the seal of the self-etching resin-based cement is comparable to those employing adhesives for sealing dentin. Similarly, De Munck et $a^{33}$ reported similar $\mu$ TBS values for Rely-X Unicem when bonded as recommended by the manufacture or when used in conjunction with phosphoric acid etching compared to conventional cement as a control.

Frankenberger et $\mathrm{a}^{34}$ however found that etchand-rinse adhesives combined with conventional luting resin cements revealed the best prognosis for the adhesive luting of glass ceramic inlays. Alsaleh and El-Mowafy ${ }^{35}$ also found that the shear bond strength of orthodontic brackets cemented with traditional etch-and-rinse cement was significantly higher than that of the group self-adhesive cements. Breschi et $\mathrm{al}^{36}$ also reported that most simplified one-step adhesives based on a similar concept to self etch resin cements were shown to be the least durable, while three-step etch-andrinse and two-step self-etch adhesives continue to demonstrate the highest performances.

Frankenberger et $\mathrm{al}^{37}$ and Frankenberger and Tay, ${ }^{38}$ who studied nanoleakage of adhesive systems, reported that, although a certain amount of nanoleakage was observed in all groups, it was more pronounced for the self-etch adhesives when compared to that of the conventional threestep adhesives. Similarly, Kubo et $\mathrm{al}^{39}$ found that conventional three-step adhesive systems tended to show less nanoleakage than the two-step and one-step adhesives. In addition, Živković ${ }^{40}$ reported that the best marginal seals were provided by Scotchbond Multi-Purpose and Syntac, thus confirming the significance that conditioning might have on the adhesive bond between the dentin and the composite material. This, in part, has been demonstrated in the present study, where the QESEM showed thicker hybrid layer formation with longer resin tags when using the Calibra resin cement compared to Rely X, which did not seem to affect the nanoleakage tendencies between the two cements.
In the present study, thermocycling did not significantly affect the degree of silver penetration at the tooth/restoration interface regardless of the resin cement type, indicating high bond durability. These results were in agreement with Dörfer et $\mathrm{al}^{41}$ and Bedran-de-Castro et al. ${ }^{23}$ Although they used different numbers of thermal cycles, they also found that thermal cycling did not affect the nanoleakage pattern.

The lack of change in the nanoleakage following the introduction of thermal stresses in this study can be attributed to the low $\mathrm{C}$-factor, since the Cerec blocks were bonded to flat dentin surfaces resulting in slight contraction stress at the bonding interface. When examining the effect of thermocycling on bond strength. Asaka et al ${ }^{25}$ found that 10000 thermal cycles had no influence on the bond strengths of single-step self-etch adhesive systems. Meanwhile, they found that raising the number of thermal cycles to 20000 decreased the bond strength significantly.

On the other hand, Holderegger et $\mathrm{al}^{42}$ found that thermocycling affected the bonding performance of tested cements. Such variation between their findings may be due to difference in the materials used, as some adhesive systems were adversely affected by thermal cycling, while others were capable of withstanding such thermal stresses.

\section{CONCLUSIONS}

Under the conditions of this in-vitro study, the following conclusions can be derived:

- One of the self-adhesive resin cements had a sealing ability that was comparable to conventional resin cement used with a separate adhesive.

- Thermo-cycling (500 cycles) did not significantly affect the durability of the tested resin cements.

\section{ACKNOWLEDGMENTS}

The authors would like to thank 3M/ESPE, Dentsply, and Bisco for materials donation.

\section{REFERENCES}

1. Bowen RL, Rapson JE, Dickson G. Hardening shrinkage and hygroscopic expansion of composite resins. J Dent Res 1982;61:654-658.

2. Feilzer AJ, De Gee AJ, Davidson CL. Setting stress in composite resin in relation to configuration of the restoration. $J$ Dent Res 1987;66:1636-1639. 
3. Davidson CL, De Gee AJ, Feilzer AJ. The competition between the composite-dentin bond strength and the polymerization contraction stress. J Dent Res 1984;63:1396-1399.

4. Causton BE, Miller B, Sefton J. The deformation of cusps by bonded posterior composite restorations. Br Dent $J$ 1985; 159:397-400.

5. Eick JD, Welch FH. Polymerization shrinkage of posterior composite resins and its possible influence on postoperative sensitivity. Quintessence Int 1986;17:103-111.

6. Feilzer AJ, De Gee AJ, Davidson CL. Curing contraction of composites and glass-ionomer cements. J Prosthet Dent 1988;59:297-300.

7. Feilzer AJ, De Gee AJ, Davidson CL. Increased wall-to-wall curing contraction in thin bonded resin layers. J Dent Res 1989;68:48-50.

8. Alani AH, Toh CG. Detection of microleakage around dental restorations: a review. Oper Dent 1997;22:173-185.

9. Hekimoglu C, Anil N, Yakin E A. Microleakage study of ceramic laminate veneers by autoradiography: effect of incisal edge preparation. J Oral Rehab 2004;31:265-270.

10. El Zohairy AA, De Gee AJ, Mohsen MM, Feilzer AJ. Microtensile bond strength testing of luting cements to prefabricated CAD/CAM ceramic and composite blocks. Dent Mater 2003; 19:575-583.

11. Van Meebeek B, De Munck J, Yoshide Y, Inoue S, Vargas M, Vijay P, Van Landuyt K, Lambrechts P, Vanherle G. Buonocore memorial lecture: Adhesion to enamel and dentin: Current status and future challenges. Oper Dent 2003;28:215-235.

12. Peumans M, Kanumilli P, De Munck J, Van Landuyt K, Lambrechts P, Van Meerbeek B. Clinical effectiveness of contemporary adhesives: a systematic review of current clinical trials. Dent Mater 2005;21:864-881.

13. Sano H, Shono T, Takatsu T, Hosoda H. Microporous dentin zone beneath resin-impregnated layer. Oper Dent 1994;19:59-64.

14. Andia-Merlin RY, Garone-Netto N, Arana-Chavez VE. SEM evaluation of the interaction between a three-step adhesive and dentin. Oper Dent 2001;26:440-444.

15. Paul SJ, Welter DA, Ghazi M, Pashley D. Nanoleakage at the dentin adhesive interface vs. u-Tensile bond strength. Oper Dent 1999;24:181-188.

16. Sano H, Takatsu T, Ciucchi B, Horner JA, Matthews WG, Pashley DH. Nanoleakage: leakage within the hybrid layer. Oper Dent 1995;20:18-25.

17. Li H, Burrow MF, Tyas MJ. Nanoleakage patterns of four dentin bonding systems. Dent Mater 2000;16:48-56.
18. Mitsui FHO, Peris AR, Cavalcanti AN, Marchi GM, Pimenta LAF. Influence of thermal and mechanical load cycling on microtensile bond strengths of total and self-etching adhesive systems. Oper Dent 2006;31:240-247.

19. Yuan Y, Shimada Y, Ichinose S, Tagami J. Qualitative analysis of adhesive interface nanoleakage using FE-SEM/EDS. Dent Mater 2007;23:561-569.

20. Yuan Y, Shimada Y, Ichinose S, Sadr A, Tagami J. Effects of dentin characteristics on interfacial nanoleakage. J Dent Res 2007;86:1001-1006.

21. Pioch T, Staehle HJ, Duschner H, Garcia-Godoy F. Nanoleakage at the composite-dentin interface: a review. Am J Dent 2001;14:252-258.

22. Ibarra G, Johnson GH, Geurtsen W, Vargas MA. Microleakage of porcelain veneer restorations bonded to enamel and dentin with a new self-adhesive resin-based dental cement. Dent Mater 2007;23:218-225.

23. Bedran-De-Castro AKB, Pereira PNR, Pimenta LAF, Thompson JY. Effect of thermal and mechanical load cycling on nanoleakage of Class II restorations. J Adhes Dent 2004;3:221-226.

24. Uno S, Abo T, Tanaka T, Sano H. In vitro sealing performance of two one-step adhesive systems in cervical cavities. J Adhes Dent 2004;6:211-219.

25. Asaka Y, Amano S, Rikuta A, Kurokawa H, Miyazaki M, Platt JA, Moore BK. Influence of thermal cycling on dentin bond strengths of single-step self-etch adhesive systems. Oper Dent 2007;32:73-80.

26. De Munck J, Van Landuyt K, Peumans M, Poitevin A, Lambrechts $P$, Braem M, Van Meerbeek B. A critical review of the durability of adhesion to tooth tissue: methods and results. J Dent Res 2005;84:118-132.

27. Tay FR, Pashley DH, Yoshiyama M. Two modes of nanoleakage expression in single-step adhesives. J Dent Res 2002;81:472-476.

28. Hashimoto M, Ohno H, Sano H, Kaga M, Oguchi H. Degradation patterns of different adhesives and bonding procedures. J Biomed Mater Res B Appl Biomater 2003;66:324-330.

29. Tay FR, King NM, Chan KM, Pashley DH. How can nanoleakage occur in self-etching adhesive systems that demineralize and infiltrate simultaneously? J Adhes Dent 2002;4:255-269.

30. Tay FR, Pashley DH, Suh BI, Carvalho RM, Itthagarun A. Single-step adhesives are permeable membranes. J Dent 2002;30:371-382.

31. Tay FR, Frankenberger R, Krejci I, Bouillaguet S, Pashley $\mathrm{DH}$, Carvalho RM, Lai CNS. Single-bottle adhesives behave as permeable membranes after polymerization: In vivo evidence. J Dent 2004;32:611-621. 
32. Behr M, Rosentritt M, Regnet T, Lang R, Handel G. Marginal adaptation in dentin of a self-adhesive universal resin cement compared with well-tried systems. Dent Mater 2004;20:191-197.

33. De Munk J, Vargas MA, Van Landuyt K, Hikita K, Lambrechts $P$, Van Meerbeek B. Bonding of an auto-adhesive luting material to enamel and dentin. Dent Mater 2004;20:963971.

34. Frankenberger R, Lohbauer U, Schaible RB, Nikolaenko SA, Naumann M. Luting of ceramic inlays in vitro: Marginal quality of self-etch and etch-and-rinse adhesives versus self-etch cements. Dent Mater 2008;24:185-191.

35. Alsaleh M, El-Mowafy 0 . Bond strength of orthodontic brackets with newself-adhesive resin cements. Am J Orthod Dentofacial Orthop 2010;137:528-533.

36. Breschi L, Mazzoni A, Ruggeri A, Cadenaro M, Di Lenarda R, Dorigo EDS. Dental adhesion review: Aging and stability of the bonded interface. Dent Mater 2008;24:90-101.

37. Frankenberger R, Pashley DH, Reich SM, Lohbauer $U$, Petschelt A, Tay FR. Characterization of resin-dentine interfaces by compressive cyclic loading. Biomaterials 2005;26:2043-2052.

38. Frankenberger R, Tay FR. Self-etch vs. etch-and-rinse adhesives: effect of thermo-mechanical fatigue loading on marginal quality of bonded resin composite restorations. Dent Mater 2005;21:397-412.

39. Kubo S, Li H, Burrow MF, Tyas MJ. Nanoleakage of dentin adhesive systems bonded to carisolv-treated dentin. Oper Dent 2002;27:387-395.

40. Živković S. Quality assessment of marginal sealing using 7 dentin adhesive systems. Quintessence Int 2000;31:423-429.

41. Dörfer CE, Staehle HJ, Wurst MW, Duschner H, Pioch T. The nanoleakage phenomenon: influence of different dentin bonding agents, thermocycling and etching time. Eur $J$ Oral Sci 2000;108:346-351.

42. Holderegger C, Sailer I, Schuhmacher C, Schläpfer R, Hämmerle C, Fischer J. Shear bond strength of resin cements to human dentin. Dent Mater 2008;24:944-950. 